This page intentionally left blank.

Designed for two-page view. 


\section{Hermann G. Matthies}

\section{WHITE NOISE ANALYSIS FOR STOCHASTIC PARTIAL DIFFERENTIAL EQUATIONS}

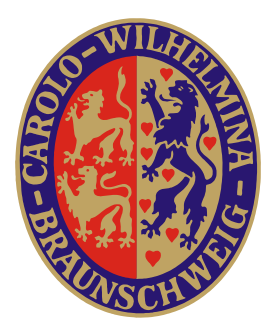

INFORMATIKBERICHT NR. 2011-03

Institute of Scientific Computing

CARL-FriedriCH-GAUSS-FAKUltäT

TeChnische Universität BraunschWeig

Brunswick, Germany 
This document was created March 2010 using $\mathrm{IAT}_{\mathrm{E}} \mathrm{X} 2_{\varepsilon}$.

Institute of Scientific Computing

Technische Universität Braunschweig Hans-Sommer-Straße 65

D-38106 Braunschweig, Germany

url: www.wire.tu-bs.de

mail: wire@tu-bs.de

Copyright (C) by Hermann G. Matthies

This work is subject to copyright. All rights are reserved, whether the whole or part of the material is concerned, specifically the rights of translation, reprinting, reuse of illustrations, recitation, broadcasting, reproduction on microfilm or in any other way, and storage in data banks. Duplication of this publication or parts thereof is permitted in connection with reviews or scholarly analysis. Permission for use must always be obtained from the copyright holder.

Alle Rechte vorbehalten, auch das des auszugsweisen Nachdrucks, der auszugsweisen oder vollständigen Wiedergabe (Photographie, Mikroskopie), der Speicherung in Datenverarbeitungsanlagen und das der Übersetzung. 


\title{
White noise analysis for stochastic partial differential equations
}

\author{
Hermann G. Matthies \\ Institute of Scientific Computing, TU Braunschweig \\ wire@tu-bs.de
}

\begin{abstract}
Stochastic partial differential equations arise when modelling uncertain phenomena. Here the emphasis is on uncertain systems where the randomness is spatial. In contrast to traditional slow computational approaches like Monte Carlo simulation, the methods described here can be orders of magnitude more efficient. These more recent methods are based on some kind stochastic Galerkin approximations, approximating the unknown quantities as functions of independent random variables, hence the name "white noise analysis". We outline the steps leading to the fully discrete equations, commenting on one possible numerical solution method. Key to many of the developments is tensor product structure of the solution, which must be exploited both theoretically and numerically. For two examples with polynomial nonlinearities the computations are shown to be quite explicit and can be performed largely analytically.
\end{abstract}

Keywords: nonlinear stochastic elliptic partial differential equations, stochastic Galerkin methods, Wiener's polynomial chaos, white noise analysis, stochastic finite elements

AMS classification: 34F05 35R60 60H35 60H15 65N30 65C05 67S05 


\section{Contents}

Contents $\quad$ v

1 Introduction $\quad 1$

2 Deterministic model problem 2

3 Stochastic model problem 3

4 Discretisation in space $\quad 5$

5 Discretisation of the probability space 5

$\begin{array}{lll}6 & \text { Solution methods } & 7\end{array}$

7 Polynomial Nonlinearities $\quad 8$

7.1 Nonlinear Diffusion . . . . . . . . . . . . . . . . . . . . 8

7.2 Stationary Navier-Stokes . . . . . . . . . . . . . . . . 10

8 Conclusion $\quad 12$

$\begin{array}{ll}\text { A Multi-Indices } & 12\end{array}$

B Hermite Polynomials 12

$\begin{array}{ll}\text { C The Hermite Algebra } & 13\end{array}$

D The Hermite Transform 14

$\begin{array}{ll}\text { References } & 16\end{array}$ 


\section{Introduction}

Oftentimes, numerical simulations of real-world systems are required even though not all parameters are exactly known. The uncertainties inherent in the model result in uncertainties in the results of numerical simulations, a fact which is often ignored in common practise. Clearly, it is desirable to quantify the uncertainties in the solution depending on the model's uncertainties.

Stochastic models are one way to quantify uncertainties. Uncertain parameters are modelled by random variables, uncertain time-dependent functions by stochastic processes, and uncertain spatial properties by random fields $[2,3,9,50]$. If the physical system is described by a partial differential equation (PDE), then the combination with the stochastic model results in a stochastic PDE (SPDE) $[18,27,11,42]$. The solution of the SPDE is a random field describing both the expected system-response and its quantitative uncertainty.

These are parametrised equations, and such parametrised equations naturally have solutions in tensor product spaces. Solution methods for such a problem range over a wide set of approaches, see [24, 30, 43, 44, 48, 23, 32, 33,34 for some developments mainly in the field of stochastic mechanics.

Next to the well-known spatial and temporal discretisation of the partial differential equation, the stochastic processes and random fields have to be discretised, and for the purpose of computation be approximated by a finite number of random variables. For computational purposes it is advantageous to describe and approximate the problem in independent random variables, a technique also known as "white noise analysis" [22, 17, 18, 19, 28].

The next step is to compute the response and its stochastic description in terms of the stochastic input. To start, we need a description of the mathematical setting which allows one to see that such stochastic models are well-posed in the sense of Hadamard essentially if the underlying deterministic model is so, and if this also holds for every possible realisation $[4,13,27,32,34,41]$. This will be briefly sketched here in Sections 2 and 3.

Solution methods [34] comprise direct integration, including Monte Carlo $[7,45]$ and its relatives, as well as deterministic integration methods such as Smolyak sparse-grid methods [14, 38, 40], stochastic collocation [6, 36, 37], and stochastic Galerkin methods [16, 31, 20, 4, 52, 26, 32, 5, 52, 51, 1, 53, 41], to name a few of the more popular ones. Here a variational framework for stochastic Galerkin (SG) methods will be given, numerical experiments may be found in the references just cited. The usual deterministic part will be summarised in Section 4, and the stochastic discretisation will be given for a simple but important kind of choice of approximating subspaces in Section 5 .

In Section 6 a very brief description of a possible numerical method for 
the solution of the fully discrete set of nonlinear equations is given, one that gives promising results $[32,33,34]$ and observes the highly structured nature of the operations on tensor product spaces.

Two examples are given in Section 7 where the nonlinearity is polynomial, in Subsection 7.1 a nonlinear diffusion, and in Subsection 7.2 the stationary Navier-Stokes equation is considered. With the properties of the Hermite algebra, given in Appendices $\mathrm{C}$ and $\mathrm{D}$, the computation of the nonlinearities can be quite explicit, with a large part of the computations performed analytically. We close with a conclusion and outlook on further work in Section 8.

\section{Deterministic model problem}

The model problem is formally one of stationary diffusion, and it is intended to serve as a motivating example on how SPDEs may arise. It may for example describe the seepage of groundwater through a porous subsurface rock / sand formation, or heat conduction in an inhomogeneous medium.

We first introduce the deterministic problem, where $\mathcal{G} \subset \mathbb{R}^{d}$ is the spatial domain of interest, $u$ is the diffusing quantity, $\boldsymbol{\kappa}$ is the diffusion tensor in the non-linear diffusion law for the flow $\boldsymbol{q}=-\boldsymbol{\kappa}(u) \nabla u$. As the diffusion tensor may depend on $u$, the problem may be nonlinear. The quantity $f$ represents sinks and sources in the domain. For simplicity we assume homogeneous Dirichlet boundary conditions. The stationary diffusion equation then is

$$
-\nabla \cdot(\boldsymbol{\kappa}(\boldsymbol{x}, \boldsymbol{u}(\boldsymbol{x})) \nabla u(x))=f(x), \quad x \in \mathcal{G} \subset \mathbb{R}^{d} .
$$

For the sake of simplicity also the conductivity tensor $\boldsymbol{\kappa}$ is represented by just a scalar field $\kappa$. None of these simplifications have any influence on what we want to show later.

For the possible solutions we choose a closed subspace of the Sobolev space $W_{p}^{1}(\mathcal{G})$, namely the completion of the compactly supported smooth functions in the $W_{p}^{1}$-norm

$$
\mathcal{U}:=\stackrel{\circ}{W}_{p}^{1}(\mathcal{G}),
$$

so that the essential Dirichlet boundary conditions are satisfied, and allow for the right-hand-side $f \in \mathcal{U}^{*} \simeq W_{q}^{-1}(\mathcal{G})$, where as usual $1 / p+1 / q=1$. To describe the diffusive process, define the generalised Nemytskii-operator $\mathrm{K}: \mathcal{U} \rightarrow \mathcal{Q}:=L_{q}\left(\mathcal{G}, \mathbb{R}^{d}\right)$ by

$$
\mathrm{K}: u(x) \mapsto\left(\varkappa(x)+c u(x)^{2}\right) \nabla u(x)=: \kappa(x, u(x)) \nabla u(x) .
$$

This is a continuous map from $\mathcal{U}=\stackrel{\circ}{W}_{p}^{1}(\mathcal{G})$ into $\mathcal{Q}=L_{q}\left(\mathcal{G}, \mathbb{R}^{d}\right)$ for $p=4$ because of the type of nonlinearity. Additionally we require $c>0, \varkappa(x)>0$ a.e., $\varkappa \in L_{\infty}(\mathcal{G})$ and $1 / \varkappa \in L_{\infty}(\mathcal{G})$. 
This makes the semilinear (linear in $v$ ) form

$$
\mathrm{a}(v, u):=\int_{\mathcal{G}} \nabla v(x) \cdot \mathrm{K}(u)(x) \mathrm{d} x
$$

hemicontinuous in $u$ and continuous in $v$, and defines a hemicontinuous nonlinear operator $\mathrm{A}: \mathcal{U} \rightarrow \mathcal{U}^{*}$ such that

$$
\forall u, v \in \mathcal{U}: \quad \mathrm{a}(v, u)=\langle\mathrm{A}(u), v\rangle_{\mathcal{U}},
$$

where $\langle\cdot, \cdot\rangle_{\mathcal{U}}$ is the duality pairing between $\mathcal{U}$ and its dual $\mathcal{U}^{*}$. If there is no danger of confusion, we will omit the index on the duality pairing.

Proposition 1. The operator A is hemicontinuous, strictly monotone and coercive. Standard arguments on monotone operators e.g. [21, 39] allow us then to conclude that under the conditions just described, the problem to find $u \in \mathcal{U}$ such that

$$
\forall v \in \mathcal{U}: \quad \mathrm{a}(v, u)=\langle\mathrm{A}(u), v\rangle=\langle f, v\rangle
$$

has a unique solution. In the linear case this reduces to the Lax-Milgram lemma.

This result shall serve as a reference of how we would like to formulate the stochastic problem in the next Section 3, namely have a well-posed problem in the sense of Hadamard. In the deterministic case it is well-known that this property of well-posedness will be inherited by the numerical approximation if it is done right.

\section{Stochastic model problem}

In the stochastic case, we want to model $\varkappa$ as well as $f$ as random fields defined over some probability space $(\Omega, \mathfrak{A}, \mathbb{P})$, where $\Omega$ is the basic probability set of elementary events, $\mathfrak{A}$ a $\sigma$-algebra of subsets of $\Omega$, and $\mathbb{P}$ a probability measure. We require additionally

$$
\varkappa(x, \omega)>0 \quad \text { a.e., } \quad\|\varkappa\|_{L_{\infty}(\mathcal{G} \times \Omega)}<\infty, \quad\|1 / \varkappa\|_{L_{\infty}(\mathcal{G} \times \Omega)}<\infty .
$$

The solution to Eq. (6) will also be a random field in that case, and we allow for that by choosing as a solution space

$$
\mathcal{W}:=\mathcal{U} \otimes \mathcal{S}
$$


where in this case we choose $\mathcal{S}=L_{p}(\Omega)$ because of the type of nonlinearity. The basic tensor product space is isomorphic to the space of finite rank linear maps $L_{q}(\Omega) \simeq \mathcal{S}^{*} \rightarrow \mathcal{U}$ which may be equipped with the Schatten- $p$-norm [46]. This is the $\ell_{p}$-norm of the sequence of singular values, and we take $\mathcal{W}$ to actually be the completion of the so normed tensor product. This is a reflexive space, just as $\mathcal{U}$, and $\mathcal{U}$ is naturally isometrically embedded via $u \mapsto u \otimes 1$ as a deterministic subspace.

We define a semilinear form a on $\mathcal{W}$ via

$$
\mathbf{a}(v, u):=\mathbb{E}(\mathrm{a}[\omega](u(x, \omega, v(x, \omega))),
$$

where $\mathbb{E}(\cdot)$ is the expectation on $\Omega$. The parameter-dependent semilinear forms are just as for the deterministic problem Eq. (4):

$$
\mathrm{a}[\omega](v, u):=\int_{\mathcal{G}} \nabla v(x, \omega) \cdot \mathbf{K}(x, \omega, u(x, \omega)) \mathrm{d} x,
$$

where the generalised Nemytskii-operator on $\mathrm{K}: \mathcal{W}=\mathcal{U} \otimes \mathcal{S} \rightarrow \mathcal{Q} \otimes \mathcal{S}^{*}$ is given by

$$
\mathbf{K}: u(x, \omega) \mapsto\left(\varkappa(x, \omega)+c u(x, \omega)^{2}\right) \cdot \nabla u(x, \omega) .
$$

Again, this defines a hemicontinuous nonlinear operator $\mathbf{A}: \mathcal{W} \rightarrow \mathcal{W}^{*}$ such that

$$
\forall u, v \in \mathcal{W}: \quad \mathbf{a}(v, u)=\langle\langle\mathbf{A}(u), v\rangle\rangle,
$$

where $\langle\langle\cdot, \cdot\rangle\rangle$ is the duality pairing between $\mathcal{W}^{*}$ and $\mathcal{W}$. Here $\mathcal{W}^{*}$ is isomorphic to the completion of $\mathcal{U}^{*} \otimes \mathcal{S}^{*}$ in the Schatten- $q$-norm.

A linear form $\mathbf{f}$ on $\mathcal{W}$ is similarly defined through its deterministic but parameter-dependent counterpart for all $v \in \mathcal{U}$

$$
\langle f(\omega), v\rangle:=\int_{\mathcal{G}} v(x) f(x, \omega) \mathrm{d} x,
$$

by $\langle\langle\mathbf{f}, w\rangle\rangle:=\mathbb{E}(\langle f(\omega), w(\omega)\rangle)$ for all $w \in \mathcal{W}$.

Proposition 2. The operator $\mathbf{A}$ is hemicontinuous, strictly monotone and coercive, and standard arguments on monotone operators (cf. Proposition 1) allow us then to conclude that the problem to find $u \in \mathcal{W}$ such that

$$
\forall v \in \mathcal{W}: \quad \mathbf{a}(v, u)=\langle\langle\mathbf{A}(u), v\rangle\rangle=\langle\langle f, v\rangle\rangle
$$

has a unique solution. In the linear case this reduces to the Lax-Milgram lemma again. 


\section{Discretisation in space}

Almost any technique may be used for the spatial discretisation, e.g. finite differences or finite elements, and we use a finite element discretisation of the region $\mathcal{G} \subset \mathbb{R}^{d}$ with a vector of ansatz-functions $\phi(x)=\left[\phi_{1}(x), \ldots, \phi_{N}(x)\right]$, e.g. $[47,10]$. We define $\mathcal{U}_{N}:=\operatorname{span}\left\{\phi_{n} \mid 1 \leq n \leq N\right\} \subset \mathcal{U}$. An ansatz for the solution in terms of $\phi(x)$ yields a semi-discretisation of Eq. (14). Similarly to the method of lines for instationary boundary value problems where the coefficients would be time-dependent, we obtain an expansion

$$
u^{\mathrm{semi}}(x, \omega)=\sum_{n=1}^{N} u_{n}(\omega) \phi_{n}(x)=\phi(x) \boldsymbol{u}(\omega),
$$

where the coefficients are random variables $\boldsymbol{u}(\omega)=\left[u_{1}(\omega), \ldots, u_{N}(\omega)\right]^{T}$.

By inserting the ansatz into the SPDE Eq. (14) and applying Galerkin conditions, a system of $N$ nonlinear stochastic equations in $\mathbb{R}^{N}$ results,

$$
\boldsymbol{A}[\omega](\boldsymbol{u}(\omega))=\boldsymbol{f}(\omega) \quad \text { for } \mathbb{P} \text {-almost all } \omega \in \Omega .
$$

Here the $n$-th equation is given by $\left.a[\omega]\left(u^{\text {semi }}(\cdot, \omega)\right), \phi_{n}\right)=:(\boldsymbol{A}[\omega](\boldsymbol{u}(\omega)))_{n}$ and $(\boldsymbol{f}(\omega))_{n}:=\left\langle f(\cdot, \omega), \phi_{n}\right\rangle$. It is worth noting that almost surely in $\omega$, the operator in Eq. (16) inherits the properties of Propositions 1 and 2 -in fact essentially uniformly in $\omega$ due to Eq. (7) - as it is a symmetric BubnovGalerkin projection onto the subspace $\mathcal{U}_{N} \otimes \mathcal{S}[47,10,32,34]$.

\section{Discretisation of the probability space}

In the following we will use a stochastic Galerkin (SG) method to fully discretise Eq. (16) [15, 16, 31, 20, 4, 52, 26, 32, 5, 1, 51, 41]. To effect the full Galerkin approximation one still has to choose ansatz functions - effectively functions of known RVs - in which to express the unknown coefficients (RVs) $u_{n}(\omega)$. We choose as ansatz functions Wiener's polynomial chaos expansion (PCE) $[25,16,31,32,34]$, i.e. multivariate Hermite polynomials $H_{\alpha}$ in Gaussian RVs. The multivariate Hermite polynomials are given in Appendix B. Reassuringly, the Cameron-Martin theorem [22, 17, 18, 19, 28] tells us that the algebra of Gaussian variables is dense in all $L_{p}(\Omega)$ with $1 \leq p<\infty$, hence in particular in $\mathcal{S}=L_{4}(\Omega)$.

For example, if we simply decide to have an approximation in $K$ Gaussian RVs with a total polynomial degree of $P$ to choose a finite basis, then one chooses a $\mathcal{A}$ as a finite subset of $\mathcal{J}:=\mathbb{N}_{0}^{(\mathbb{N})}$, the set of all finite non-negative 
integer sequences, i.e. of multi-indices, see Appendix A.

$$
\mathcal{A}=\left\{\alpha=\left(\alpha_{1}, \ldots, \alpha_{K}, \ldots\right) \in \mathcal{J} \mid \alpha_{k}=0 \text { for } k>K, \text { and }|\alpha|_{1}<P\right\},
$$

where the cardinality of $\mathcal{A}$ is

$$
A:=|\mathcal{A}|=\frac{(K+P) !}{K ! P !} .
$$

Although the set $\mathcal{A}$ is finite and $\mathcal{J}$ is countable, there is no natural order on it; we therefore do not impose one at this point. The determination of $\mathcal{A}$ via $K$ and $P$ as above is in many cases too crude, not least because the cardinality changes very unevenly with changing $K$ and $M$. More elaborate ways to define $\mathcal{A}$ have to be employed using different functionals than just the $\ell_{1}$-norm.

As ansatz in the probabilistic or stochastic space we take

$$
\boldsymbol{u}(\omega)=\sum_{\alpha \in \mathscr{A}} \boldsymbol{u}^{\alpha} H_{\alpha}(\boldsymbol{\theta}(\omega))
$$

with $\boldsymbol{u}^{\alpha}:=\left[u_{1}^{\alpha}, \ldots, u_{N}^{\alpha}\right]^{T}$. Through the discretisation the stochastic space $\mathcal{S}$ has been replaced by a subspace $\mathcal{S}_{\mathcal{A}}:=\operatorname{span}\left\{H_{\alpha} \mid \alpha \in \mathcal{A}\right\}$.

The Bubnov-Galerkin method applied to Eq. (16) with the ansatz Eq. (17) requires that the weighted residuals vanish:

$$
\forall \beta \in \mathcal{A}: \quad \mathbb{E}\left(\left[\boldsymbol{f}(\omega)-\boldsymbol{A}[\omega]\left(\sum_{\alpha \in \mathcal{A}} \boldsymbol{u}^{\alpha} H_{\alpha}(\boldsymbol{\theta}(\omega))\right)\right] H_{\beta}(\omega)\right)=0 .
$$

This may be concisely written - with quantities in the fully discrete space $\mathbb{R}^{N} \otimes \mathbb{R}^{A}$ denoted by an upright bold font-as

$$
\mathbf{r}(\mathbf{u}):=\mathbf{f}-\mathbf{A}(\mathbf{u})=0, \text { or } \mathbf{A}(\mathbf{u})=\mathbf{f},
$$

where $(\mathbf{f})_{n}^{\beta}:=\mathbb{E}\left((\boldsymbol{f}(\omega))_{n} H_{\beta}(\omega)\right), \mathbf{u}:=\left(u_{n}^{\alpha}\right)$, and

$$
(\mathbf{A}(\mathbf{u}))_{n}^{\beta}=\mathbb{E}\left(\left(\boldsymbol{A}[\omega]\left(\sum_{\alpha \in \mathcal{A}} \boldsymbol{u}^{\alpha} H_{\alpha}(\omega)\right)\right)_{n} H_{\beta}(\omega)\right) .
$$

A quantity like $\mathbf{u}$ may be thought of as an array of numbers $\left(u_{n}^{\alpha}\right)$, exploiting the isomorphy $\mathbb{R}^{N} \otimes \mathbb{R}^{A} \simeq \mathbb{R}^{N \times A}$, or as an abstract tensor $\sum_{\alpha} \boldsymbol{u}^{\alpha} \otimes \boldsymbol{e}_{\alpha}$, where the $\boldsymbol{e}_{\alpha}$ are the canonical unit vectors in $\mathbb{R}^{A}$, or-in a purely linear algebra fashion - regard the symbol $\otimes$ consistently as a Kronecker product. It may be noted that Eq. (19) are $A \times N$ equations, and the system Eq. (19) inherits the properties of Propositions 1 and 2 as it is a symmetric Bubnov-Galerkin projection onto the finite dimensional subspace $\mathcal{W}_{N, \mathcal{A}}:=\mathcal{U}_{N} \otimes \mathcal{S}_{\mathcal{A}}$. 
Proposition 3. Convergence of the full Galerkin approximation [32, 34] with coefficients the solution from Eq. (19)

$$
u^{\mathrm{f}}(x, \omega):=\sum_{\alpha}\left(\phi(x) \boldsymbol{u}^{\alpha}\right) H_{\alpha}(\boldsymbol{\theta}(\omega))=\sum_{n, \alpha} u_{n}^{\alpha} \phi_{n}(x) H_{\alpha}(\boldsymbol{\theta}(\omega)),
$$

to the solution of the SPDE $u$ from Eq. (14) with increasing densly filling subspaces $\mathcal{W}_{N, \mathcal{A}} \subseteq \mathcal{W}$ may be established with Céa's lemma [47, 10] as being quasi-optimal:

$$
\left\|u-u^{\mathrm{f}}\right\|_{\mathcal{W}} \leq C \inf _{v \in \mathcal{W}_{N, \mathcal{A}}}\|u-v\|_{\mathcal{W}}
$$

For better convergence estimates, one would need results on the regularity of the solution $u$ to Eq. (14). For norms weaker than the Schatten- $p$-norm used in Eq. (21), one may take the results in [8], these show the benefit of not only increasing the polynomial degree, but also the total number $K$ of RVs used in the approximation.

\section{Solution methods}

We may solve the nonlinear system Eq. (19) by the BFGS method with linesearches, e.g. cf. [29, 12]. In every iteration a correction of the current iterate $\mathbf{u}_{k}$ is computed as

$$
\begin{aligned}
\mathbf{u}_{k+1}-\mathbf{u}_{k} & =-\mathbf{H}_{k} \mathbf{r}\left(\mathbf{u}_{k}\right), \\
\mathbf{H}_{k} & =\mathbf{H}_{0}+\sum_{j=1}^{k}\left(r_{j} \mathbf{p}_{j} \otimes \mathbf{p}_{j}+s_{j} \mathbf{q}_{j} \otimes \mathbf{q}_{j}\right) .
\end{aligned}
$$

The tensors $\mathbf{p}_{j}, \mathbf{q}_{j}$ and the scalars $r_{j}, s_{j}$ are results of the previous iterations of the BFGS method, cf. [29, 12]. A preconditioner or initial $\mathbf{H}_{0}$ is necessary in order to obtain good convergence. Most preconditioners have the form $\mathbf{H}_{0}=\boldsymbol{M} \otimes \boldsymbol{\Xi}$ with matrices $\boldsymbol{M} \in \mathbb{R}^{N \times N}$ and $\boldsymbol{\Xi} \in \mathbb{R}^{A \times A}[32,49]$, and hence display a typical tensor-product structure. One may note that Eq. (22) is an iteration on tensors, and that the update to the operator is also in form of a rank-2-tensor. Needless to say that in actual computations, neither in Eq. (22) nor anywhere else are the tensor products like in Eq. (23) actually formed [29]. This would completely destroy the very sparse nature of the computations, but rather the components are always only used in the form of an operator and stored separately [54]. 


\section{Polynomial Nonlinearities}

While the development of the previous sections gives a general avenue to approach not only the formulation and discretisation of nonlinear SPDEs, but also the actual numerical solution process for the discrete solution, in many cases one can be more specific. Often the nonlinearity is just a polynomial in the solution (or may be represented by a power series in the solution), e.g. the Navier-Stokes Equation, where the nonlinearity is just quadratic. For this it is advantageous to have a direct representation of polynomials of random variables.

In Appendices $\mathrm{C}$ and $\mathrm{D}$ it is shown how to treat polynomial nonlinearities in terms of the Hermite-algebra and Hermite transform, and that will be employed here.

Computationally we will represent random variables $r_{1}, r_{2}, \ldots$ by the sequence of their PCE-coefficients $\left(\rho_{1}\right)=\mathscr{H}\left(\mathrm{r}_{1}\right),\left(\rho_{2}\right)=\mathscr{H}\left(\mathrm{r}_{2}\right)$ etc., see Appendix D. This then allows us to express products of two - see Eq. (65) and Eq. (66), or more random variables similarly to Eq. (67) - all with the help of the Hermite transform.

\subsection{Nonlinear Diffusion}

Let us take a look at the introductory example of a nonlinear diffusion equation Eq. (1) with the specific nonlinearity Eq. (11). After semi-discretisation the Eq. (16) may be written as

$$
\boldsymbol{A}[\omega]\left(\boldsymbol{u}(\omega)=\left(\boldsymbol{K}_{0}(\omega)+\boldsymbol{K}_{c}(\boldsymbol{u}(\omega))\right) \boldsymbol{u}(\omega)=\boldsymbol{f}(\omega),\right.
$$

where $\boldsymbol{u}(\omega)$ and $\boldsymbol{f}(\omega)$ are as before, and almost as a usual stiffness matrix

$$
\left(\boldsymbol{K}_{0}(\omega)\right)_{n, m}:=\int_{\mathcal{G}} \nabla \phi_{n}(x) \cdot \varkappa(x, \omega) \nabla \phi_{m}(x) \mathrm{d} x,
$$

and

$$
\left(\boldsymbol{K}_{c}(\boldsymbol{u}(\omega))\right)_{n, m}:=\int_{\mathcal{G}} \nabla \phi_{n}(x) \cdot c\left(u^{\mathrm{f}}(x, \omega)\right)^{2} \nabla \phi_{m}(x) \mathrm{d} x,
$$

with $u^{\mathrm{f}}(x, \omega):=\sum_{\alpha}\left(\phi(x) \boldsymbol{u}^{\alpha}\right) H_{\alpha}(\boldsymbol{\theta}(\omega))$. This quantity may also be expressed with $\mathbf{u}=\left[u_{n}^{\alpha}\right]$ for later use as $u^{\mathrm{f}}(\mathbf{u}):=u^{\mathrm{f}}(x, \omega)=\sum_{n, \alpha} u_{n}^{\alpha} \phi_{n}(x) H_{\alpha}(\boldsymbol{\theta}(\omega))$. By denoting $u_{\alpha}^{\mathrm{f}}(x):=\boldsymbol{\phi}(x) \boldsymbol{u}^{\alpha}=\sum_{n} u_{n}^{\alpha} \phi_{n}(x)$, we recognise these coefficients to be the Hermite transform $\mathscr{H}\left(u^{\mathrm{f}}(x, \omega)\right)=\left(u_{\alpha}^{\mathrm{f}}(x)\right)_{\alpha \in \mathcal{J}}$, see Appendix D. From this and with the notation $\left(u_{i}\right)=\left(\ldots, u_{i}^{\alpha}, \ldots\right)$ one sees that the PCE 
of $\left(u^{\mathrm{f}}(x, \omega)\right)^{2}$ is

$$
\begin{aligned}
\left(u^{\mathrm{f}}(\mathbf{u})\right)^{2}=\left(u^{\mathrm{f}}(x, \omega)\right)^{2}= & \mathscr{H}^{-1}\left(\mathbf{C}_{2}\left(\left(u_{\alpha}^{\mathrm{f}}(x)\right),\left(u_{\alpha}^{\mathrm{f}}(x)\right)\right)\right)= \\
& \sum_{\gamma}\left[\sum_{i, j} \phi_{i}(x)\left(\left(u_{i}\right) \boldsymbol{C}_{2}^{\gamma}\left(u_{j}\right)\right) \phi_{j}(x)\right] H_{\gamma}(\boldsymbol{\theta}(\omega)) .
\end{aligned}
$$

There are different ways of going on from here, the simplest seems to be to set in Eq. (25) $\boldsymbol{K}_{0}(\omega)=\sum_{\gamma} H_{\gamma}(\boldsymbol{\theta}(\omega)) \boldsymbol{K}_{0}^{\gamma}$, with $\boldsymbol{K}_{0}^{\gamma}:=\mathbb{E}\left(H_{\gamma} \boldsymbol{K}_{0}\right) / \gamma$ !, as the $H_{\gamma}$ are orthogonal. For Eq. (26) this looks just as simple, setting $\boldsymbol{K}_{c}(\boldsymbol{u}(\omega))=$ $\sum_{\gamma} H_{\gamma}(\boldsymbol{\theta}(\omega)) \boldsymbol{K}_{c}^{\gamma}\left(u^{\mathrm{f}}\right)$ with $\boldsymbol{K}_{c}^{\gamma}\left(u^{\mathrm{f}}\right):=\mathbb{E}\left(H_{\gamma}(\boldsymbol{\theta}(\omega)) \boldsymbol{K}_{c}(\boldsymbol{u}(\omega))\right) / \gamma$ !. The terms in the last expression may be facilitated with Eq. (27), so that

$$
\left(\boldsymbol{K}_{c}^{\gamma}\left(u^{\mathrm{f}}\right)\right)_{n, m}=\int_{\mathcal{G}} \nabla \phi_{n}(x) c\left[\sum_{i, j} \phi_{i}(x)\left(\left(u_{i}\right) \boldsymbol{C}_{2}^{\gamma}\left(u_{j}\right)\right) \phi_{j}(x)\right] \nabla \phi_{m}(x) \mathrm{d} x .
$$

Both matrices now have a PCE.

Using these PCEs when computing the terms of Eq. (19) with the help Eq. (18), we obtain

$$
\left(\mathbf{K}_{0}+\mathbf{K}_{c}\left(u^{\mathrm{f}}(\mathbf{u})\right)\right) \mathbf{u}=\mathbf{f},
$$

where $\mathbf{f}$ and $\mathbf{u}$ are as before in Eq. (19). For $\mathbf{K}_{0}$ the Galerkin projections in Eq. (18) result in

$$
\begin{aligned}
\left(\mathbf{K}_{0}\right)_{\alpha, \beta} & :=\sum_{\gamma} \mathbb{E}\left(H_{\alpha} H_{\gamma} H_{\beta}\right) \boldsymbol{K}_{0}^{\gamma}=: \sum_{\gamma} \boldsymbol{\Delta}_{\alpha, \beta}^{\gamma} \boldsymbol{K}_{0}^{\gamma}, \text { with } \\
\boldsymbol{\Delta}_{\alpha, \beta}^{\gamma} & :=\mathbb{E}\left(H_{\alpha} H_{\gamma} H_{\beta}\right)=c_{\alpha, \beta}^{\gamma} \gamma !(\text { see Appendix } \mathrm{C}) .
\end{aligned}
$$

This can be written as a tensor product

$$
\mathbf{K}_{0}=\sum_{\gamma} \boldsymbol{K}_{0}^{\gamma} \otimes \Delta^{\gamma}
$$

Similarly, for $\mathbf{K}_{c}$ the Galerkin projections in Eq. (18) result in

$$
\left(\mathbf{K}_{c}\right)_{\alpha, \beta}\left(u^{\mathrm{f}}(\mathbf{u})\right):=\sum_{\gamma} \boldsymbol{\Delta}_{\alpha, \beta}^{\gamma} \boldsymbol{K}_{c}^{\gamma}\left(u^{\mathrm{f}}\right) .
$$

This can again be written as a tensor product

$$
\mathbf{K}_{c}\left(u^{\mathrm{f}}(\mathbf{u})\right)=\sum_{\gamma} \boldsymbol{K}_{c}^{\gamma}\left(u^{\mathrm{f}}\right) \otimes \boldsymbol{\Delta}^{\gamma} .
$$


All the terms of the nonlinear Eq. (29) have now explicitly computed, most of them purely analytically. This shows the power of the Hermite algebra calculus for such polynomial nonlinearities, giving the explicit form of Eq. (29) as

$$
\left(\sum_{\gamma} \boldsymbol{K}_{0}^{\gamma} \otimes \boldsymbol{\Delta}^{\gamma}+\sum_{\gamma} \boldsymbol{K}_{c}^{\gamma}\left(u^{\mathrm{f}}(\mathbf{u})\right) \otimes \boldsymbol{\Delta}^{\gamma}\right) \mathbf{u}=\mathbf{f} .
$$

One should note that, regarding the discussion following Eq. (19), the operation $\otimes$ has to be interpreted according to the context. If $\boldsymbol{H} \in \mathbb{R}^{N \times N}$, $\boldsymbol{\Psi} \in \mathbb{R}^{A \times A}, \boldsymbol{h} \in \mathbb{R}^{N}$, and $\boldsymbol{\psi} \in \mathbb{R}^{A}$, then the operator $\boldsymbol{H} \otimes \boldsymbol{\Psi}$ acts on the tensor $\boldsymbol{h} \otimes \boldsymbol{\psi}$ as

$$
(\boldsymbol{H} \otimes \boldsymbol{\Psi}) \boldsymbol{h} \otimes \boldsymbol{\psi}:=(\boldsymbol{H} \boldsymbol{h}) \otimes(\boldsymbol{\Psi} \boldsymbol{\psi}),
$$

and is extended by continuity to the whole space. If, as already mentioned, the symbol $\otimes$ is consistently interpreted as a Kronecker product, one gets a fully linear algebra like description, whereas interpreting $\mathbf{u}$ as a matrix $\boldsymbol{U}=\left[u_{n}^{\alpha}\right]$, the operator acts as $\boldsymbol{H} \boldsymbol{U} \boldsymbol{\Psi}^{T}$.

\subsection{Stationary Navier-Stokes}

Let us take as another example the stationary incompressible Navier-Stokes equation (with appropriate boundary conditions), where the nonlinearity is quadratic:

$$
v \cdot \nabla v-\frac{1}{\operatorname{Re}} \nabla^{2} v+\nabla p=g, \text { and } \nabla \cdot v=0,
$$

where $v(x)$ is the velocity vector at position $x$, the pressure at $x$ is given by $p(x)$, the body force per unit mass is $g(x)$, and Re is the Reynolds number. Assuming that boundary conditions, or initial conditions, or right hand side $g$ are uncertain, we model the response as random fields $v(x, \omega)$ and $p(x, \omega)$.

In a discretised version, the Eq. (36) will look like

$$
\boldsymbol{N}(\boldsymbol{v}, \boldsymbol{v})+\boldsymbol{K} \boldsymbol{v}+\boldsymbol{B} \boldsymbol{p}=\boldsymbol{g}, \text { and } \boldsymbol{B}^{T} \boldsymbol{v}=\mathbf{0},
$$

where the type of discretisation is not really important for the formulation of the stochastic response. The bilinear operator $\boldsymbol{N}(\cdot, \cdot)$ comes from the nonlinear convective acceleration term, $\boldsymbol{K}$ is the matrix corresponding to the diffusive part, and $\boldsymbol{B}$ is a discrete gradient; $\boldsymbol{v}$ and $\boldsymbol{p}$ are the vectors for the discrete representation of the velocity $v$ and pressure $p$.

Remark 1. It may be injected here, that if the Reynolds number-or rather the viscosity as the density is constant for an incompressible flow-were to be 
regarded as random field, then the matrix $\boldsymbol{K}$ in Eq. (37) would be a random matrix like $\boldsymbol{K}_{0}$ in Eq. (24) with a corresponding PCE.

Expressing the quantities involved in their PCE

$$
\begin{aligned}
& \boldsymbol{v}(\boldsymbol{\theta}(\omega))=\sum_{\alpha \in \mathcal{A}} \boldsymbol{v}^{\alpha} H_{\alpha}(\boldsymbol{\theta}(\omega)), \\
& \boldsymbol{p}(\boldsymbol{\theta}(\omega))=\sum_{\beta \in \mathcal{A}} \boldsymbol{p}^{\beta} H_{\beta}(\boldsymbol{\theta}(\omega)), \\
& \boldsymbol{g}(\boldsymbol{\theta}(\omega))=\sum_{\gamma \in \mathcal{A}} \boldsymbol{g}^{\gamma} H_{\gamma}(\boldsymbol{\theta}(\omega)),
\end{aligned}
$$

one obtains with the help of Appendices C and D

$$
\sum_{\beta, \gamma \in \mathcal{A}} \boldsymbol{N}\left(\boldsymbol{v}^{\beta}, \boldsymbol{v}^{\gamma}\right) H_{\beta} H_{\gamma}+\sum_{\alpha \in \mathcal{A}} \boldsymbol{K} \boldsymbol{v}^{\alpha} H_{\alpha}+\sum_{\alpha \in \mathcal{A}} \boldsymbol{B} \boldsymbol{p}^{\alpha} H_{\alpha}=\sum_{\alpha \in \mathcal{A}} \boldsymbol{g}^{\alpha} H_{\alpha}
$$

and

$$
\sum_{\alpha \in \mathcal{A}} \boldsymbol{B}^{T} \boldsymbol{v}^{\alpha} H_{\alpha}=\mathbf{0}
$$

With the help of Eq. (57), the nonlinear term in Eq. (41) can be rewritten as

$$
\sum_{\beta, \gamma \in \mathcal{A}} \boldsymbol{N}\left(\boldsymbol{v}^{\beta}, \boldsymbol{v}^{\gamma}\right) H_{\beta} H_{\gamma}=\sum_{\alpha}\left(\sum_{\beta, \gamma} c_{\beta \gamma}^{\alpha} \boldsymbol{N}\left(\boldsymbol{v}^{\beta}, \boldsymbol{v}^{\gamma}\right)\right) H_{\alpha}
$$

Inserting this into Eq. (41) and projecting onto each $H_{\alpha}$ gives

$$
\begin{array}{r}
\forall \alpha \in \mathcal{A}: \quad \sum_{\beta, \gamma} c_{\beta \gamma}^{\alpha} \boldsymbol{N}\left(\boldsymbol{v}^{\beta}, \boldsymbol{v}^{\gamma}\right)+\boldsymbol{K} \boldsymbol{v}^{\alpha}+\boldsymbol{B} \boldsymbol{p}^{\alpha}=\boldsymbol{g}^{\alpha}, \\
\text { and } \boldsymbol{B}^{T} \boldsymbol{v}^{\alpha}=\mathbf{0 .}
\end{array}
$$

Using tensor products $\mathbf{v}$ and $\mathbf{p}$ as before, and defining in the matrix representation

$$
\mathbf{N}(\mathbf{v}, \mathbf{v})=\left[\ldots, \sum_{\beta, \gamma} c_{\beta \gamma}^{\alpha} \mathbf{N}\left(\boldsymbol{v}^{\beta}, \boldsymbol{v}^{\gamma}\right), \ldots\right],
$$

this may be succinctly written as

$$
\begin{aligned}
\mathbf{N}(\mathbf{v}, \mathbf{v})+(\boldsymbol{K} \otimes \boldsymbol{I}) \mathbf{v}+(\boldsymbol{B} \otimes \boldsymbol{I}) \mathbf{p} & =\mathbf{g}, \\
\text { and }\left(\boldsymbol{B}^{T} \otimes \boldsymbol{I}\right) \mathbf{v} & =\mathbf{0} .
\end{aligned}
$$

This is an explicit PCE representation of the nonlinear stationary incompressible Navier-Stokes equation, making the Hermite-algebra calculus quite explicit. Observe that all high-dimensional integrations were done analytically. 


\section{Conclusion}

We have tried to provide a short introduction to nonlinear SPDEs and stochastic Galerkin methods based on white noise analysis. But the computational effort is often still very high even though there may be tremendous gains compared to the ubiquitous Monte Carlo method. The references mentioned in the introduction contain many interesting directions how the computational burden may be alleviated through adaptivity and model reduction or reduced order models. Some recent references to this kind of work may be found for example in $[34,35]$.

\section{A Multi-Indices}

In the above formulation, the need for multi-indices of arbitrary length arises. Formally they may be defined by

$$
\alpha=\left(\alpha_{1}, \ldots, \alpha_{\jmath}, \ldots\right) \in \mathcal{J}:=\mathbb{N}_{0}^{(\mathbb{N})},
$$

which are sequences of non-negative integers, only finitely many of which are non-zero. As by definition $0 !:=1$, the expressions

$$
|\alpha|_{1}:=\sum_{\jmath=1}^{\infty} \alpha_{\jmath} \quad \text { and } \quad \alpha !:=\prod_{\jmath=1}^{\infty} \alpha_{\jmath} !
$$

are well defined for $\alpha \in \mathcal{J}$.

\section{B Hermite Polynomials}

As there are different ways to define - and to normalise - the Hermite polynomials, a specific way has to be chosen. In applications with probability theory it seems most advantageous to use the following definition [22, 17, 18, 19, 28]:

$$
h_{k}(t):=(-1)^{k} e^{t^{2} / 2}\left(\frac{\mathrm{d}}{\mathrm{d} t}\right)^{k} e^{-t^{2} / 2} ; \quad \forall t \in \mathbb{R}, k \in \mathbb{N}_{0},
$$

where the coefficient of the highest power of $t$ - which is $t^{k}$ for $h_{k}$-is equal to unity.

The first five polynomials are:

$$
\begin{aligned}
& h_{0}(t)=1, \quad h_{1}(t)=t, \quad h_{2}(t)=t^{2}-1, \\
& h_{3}(t)=t^{3}-3 t, \quad h_{4}(t)=t^{4}-6 t^{2}+3 .
\end{aligned}
$$


The recursion relation for these polynomials is

$$
h_{k+1}(t)=t h_{k}(t)-k h_{k-1}(t) ; \quad k \in \mathbb{N} .
$$

These are orthogonal polynomials w.r.t standard Gaussian probability measure $\Gamma$, where $\Gamma(\mathrm{d} t)=(2 \pi)^{-1 / 2} e^{-t^{2} / 2} \mathrm{~d} t$ - the set $\left\{h_{k}(t) / \sqrt{k !} \mid k \in \mathbb{N}_{0}\right\}$ forms a complete orthonormal system $(\mathrm{CONS})$ in $L_{2}(\mathbb{R}, \Gamma)$ - as the Hermite polynomials satisfy

$$
\int_{-\infty}^{\infty} h_{m}(t) h_{n}(t) \Gamma(\mathrm{d} t)=n ! \delta_{n, m} .
$$

Multi-variate Hermite polynomials will be defined right away for an infinite number of variables, i.e. for $\boldsymbol{t}=\left(t_{1}, t_{2}, \ldots, t_{\jmath}, \ldots\right) \in \mathbb{R}^{\mathbb{N}}$, the space of all sequences. For $\alpha=\left(\alpha_{1}, \ldots, \alpha_{\jmath}, \ldots\right) \in \mathcal{J}$ remember that except for a finite number all other $\alpha_{\jmath}$ are zero; hence in the definition of the multi-variate Hermite polynomial

$$
H_{\alpha}(\boldsymbol{t}):=\prod_{\jmath=1}^{\infty} h_{\alpha_{\jmath}}\left(t_{\jmath}\right) ; \quad \forall \boldsymbol{t} \in \mathbb{R}^{\mathbb{N}}, \alpha \in \mathcal{J},
$$

except for finitely many factors all others are $h_{0}$, which equals unity, and the infinite product is really a finite one and well defined.

The space $\mathbb{R}^{\mathbb{N}}$ can be equipped with a Gaussian (product) measure [22, $17,18,19,28]$, again denoted by $\Gamma$. Then the set $\left\{H_{\alpha}(\boldsymbol{t}) / \sqrt{\alpha !} \mid \alpha \in \mathcal{J}\right\}$ is a CONS in $L_{2}\left(\mathbb{R}^{\mathbb{N}}, \Gamma\right)$ as the multivariate Hermite polynomials satisfy

$$
\int_{\mathbb{R}^{\mathbb{N}}} H_{\alpha}(\boldsymbol{t}) H_{\beta}(\boldsymbol{t}) \Gamma(\mathrm{d} \boldsymbol{t})=\alpha ! \delta_{\alpha \beta},
$$

where the Kronecker symbol is extended to $\delta_{\alpha \beta}=1$ in case $\alpha=\beta$ and zero otherwise.

\section{The Hermite Algebra}

Consider first the usual univariate Hermite polynomials $\left\{h_{k}\right\}$ as defined in Appendix B, Eq. (50). As the univariate Hermite polynomials are a linear basis for the polynomial algebra, i.e. every polynomial can be written as linear combination of Hermite polynomials, this is also the case for the product of two Hermite polynomials $h_{k} h_{\ell}$, which is clearly also a polynomial:

$$
h_{k}(t) h_{\ell}(t)=\sum_{n=|k-\ell|}^{k+\ell} c_{k \ell}^{n} h_{n}(t),
$$


where $n$ is an index, not an exponent. The coefficients are only non-zero [28] for integer $g=(k+\ell+n) / 2 \in \mathbb{N}$ and if $g \geq k \wedge g \geq \ell \wedge g \geq n$. They can be explicitly given

$$
c_{k \ell}^{n}=\frac{k ! \ell !}{(g-k) !(g-\ell) !(g-n) !},
$$

and are called the structure constants of the univariate Hermite algebra.

For the multivariate Hermite algebra, analogous statements hold [28]:

$$
H_{\alpha}(\boldsymbol{t}) H_{\beta}(\boldsymbol{t})=\sum_{\gamma} c_{\alpha \beta}^{\gamma} H_{\gamma}(\boldsymbol{t})
$$

with the multivariate structure constants

$$
c_{\alpha \beta}^{\gamma}=\prod_{\jmath=1}^{\infty} c_{\alpha_{\jmath} \beta_{\jmath}}^{\gamma_{\jmath}},
$$

defined in terms of the univariate structure constants Eq. (56).

From this it is easy to see that

$$
\mathbb{E}\left(H_{\alpha} H_{\beta} H_{\gamma}\right)=\mathbb{E}\left(H_{\gamma} \sum_{\varepsilon} c_{\alpha \beta}^{\varepsilon} H_{\varepsilon}\right)=c_{\alpha \beta}^{\gamma} \gamma ! .
$$

Products of more than two Hermite polynomials may be computed recursively, we here look at triple products as an example, using Eq. (57):

$$
H_{\alpha} H_{\beta} H_{\delta}=\left(\sum_{\gamma} c_{\alpha \beta}^{\gamma} H_{\gamma}\right) H_{\delta}=\sum_{\varepsilon}\left(\sum_{\gamma} c_{\gamma \delta}^{\varepsilon} c_{\alpha \beta}^{\gamma}\right) H_{\varepsilon} .
$$

\section{The Hermite Transform}

A variant of the Hermite transform maps a random variable onto the set of expansion coefficients of the PCE [18]. Any random variable which may be represented with a $\mathrm{PCE}$

$$
r(\omega)=\sum_{\alpha \in \mathcal{J}} \varrho^{\alpha} H_{\alpha}(\boldsymbol{\theta}(\omega))
$$

is mapped onto

$$
\mathscr{H}(\mathrm{r}):=\left(\varrho^{\alpha}\right)_{\alpha \in \mathcal{J}}=(\varrho) \in \mathbb{R}^{\mathcal{J}} .
$$


On the other hand, from a sequence indexed by $\mathcal{J}$, as a mapping $\rho: \mathcal{J} \rightarrow$ $\mathbb{R}: \alpha \mapsto \rho^{\alpha}$, one may obtain the random variable

$$
\mathscr{H}^{-1}((\rho))=\mathscr{H}^{-1}\left(\left(\rho^{\alpha}\right)_{\alpha \in \mathcal{J}}\right):=\sum_{\alpha \in \mathcal{J}} \rho^{\alpha} H_{\alpha}
$$

which defines the inverse Hermite transform.

These sequences may be seen also as the coefficients of power series in infinitely many complex variables $\boldsymbol{z} \in \mathbb{C}^{\mathbb{N}}$, namely by

$$
\sum_{\alpha \in \mathcal{J}} \varrho^{\alpha} z^{\alpha}
$$

where $\boldsymbol{z}^{\alpha}:=\prod_{\jmath} z_{\jmath}^{\alpha_{J}}$. This is the original definition of the Hermite transform [18].

It can be used to easily compute the Hermite transform of the ordinary product like in Eq. (57), as

$$
\mathscr{H}\left(H_{\alpha} H_{\beta}\right)=\left(c_{\alpha \beta}^{\gamma}\right)_{\gamma \in \mathcal{J}} .
$$

With the structure constants Eq. (58) one defines the matrices $\boldsymbol{C}_{2}^{\gamma}:=\left(c_{\alpha \beta}^{\gamma}\right)$ with indices $\alpha$ and $\beta$. The Hermite transform of the product of two random variables $\mathrm{r}_{1}(\omega)=\sum_{\alpha \in \mathcal{J}} \varrho_{1}^{\alpha} H_{\alpha}(\theta)$ and $\mathrm{r}_{2}(\omega)=\sum_{\beta \in \mathcal{J}} \varrho_{2}^{\beta} H_{\beta}(\boldsymbol{\theta})$ is hence

$$
\left.\mathscr{H}\left(\mathrm{r}_{1} \mathrm{r}_{2}\right)=\left(\left(\varrho_{1}\right) \boldsymbol{C}_{2}^{\gamma}\left(\varrho_{2}\right)^{T}\right)\right)_{\gamma \in \mathcal{J}}
$$

Each coefficient is a bilinear form in the coefficient sequences of the factors, and the collection of all those bilinear forms $\mathbf{C}_{2}=\left(\boldsymbol{C}_{2}^{\gamma}\right)_{\gamma \in \mathcal{J}}$ is a bilinear mapping that maps the coefficient sequences of $r_{1}$ and $r_{2}$ into the coefficient sequence of the product

$$
\mathscr{H}\left(\mathrm{r}_{1} \mathrm{r}_{2}\right)=: \mathbf{C}_{2}\left(\left(\varrho_{1}\right),\left(\varrho_{2}\right)\right)=\mathbf{C}_{2}\left(\mathscr{H}\left(\mathrm{r}_{1}\right), \mathscr{H}\left(\mathrm{r}_{2}\right)\right) .
$$

Products of more than two random variables may now be defined recursively through the use of associativity. e.g. $r_{1} r_{2} r_{3} r_{4}=\left(\left(\left(r_{1} r_{2}\right) r_{3}\right) r_{4}\right)$ :

$$
\begin{aligned}
\forall k>2: \quad \mathscr{H}\left(\prod_{\jmath=1}^{k} \mathrm{r}_{\jmath}\right):=\mathbf{C}_{k}\left(\left(\varrho_{1}\right),\left(\varrho_{2}\right), \ldots,\left(\varrho_{k}\right)\right):= \\
\mathbf{C}_{k-1}\left(\mathbf{C}_{2}\left(\left(\varrho_{1}\right),\left(\varrho_{2}\right)\right),\left(\varrho_{3}\right) \ldots,\left(\varrho_{k}\right)\right) .
\end{aligned}
$$

Each $\mathbf{C}_{k}$ is again composed of a sequence of $k$-linear forms $\left\{\boldsymbol{C}_{k}^{\gamma}\right\}_{\gamma \in \mathcal{J}}$, which define each coefficient of the Hermite transform of the $k$-fold product. 


\section{References}

[1] S. Acharjee and N. Zabaras: A non-intrusive stochastic Galerkin approach for modeling uncertainty propagation in deformation processes. Computers \& Structures 85 (2007) 244-254.

[2] R. J. Adler: The Geometry of Random Fields. John Wiley \& Sons, Chichester, 1981.

[3] R. J. Adler and J. E. Taylor: Random Fields and Geometry. SpringerVerlag, Berlin, 2007.

[4] I. Babuška, R. Tempone, and G. E. Zouraris: Galerkin finite element approximations of stochastic elliptic partial differential equations. SIAM J. Num. Anal. 42 (2004) 800-825.

[5] I. Babuška, R. Tempone and G. E. Zouraris: Solving elliptic boundary value problems with uncertain coefficients by the finite element method: the stochastic formulation. Comp. Meth. Appl. Mech. Engrg. 194 (2005) 1251-1294.

[6] I. Babuška, F. Nobile and R. Tempone: A stochastic collocation method for elliptic partial differential equations with random input data. SIAM J. Num. Anal. 45 (2007) 1005-1034.

[7] R. E. Caflisch: Monte Carlo and quasi-Monte Carlo methods. Acta Numerica 7 (1998) 1-49.

[8] Y. Cao: On the rate of convergence of Wiener-Itô expansion for generalized random variables. Stochastics 78 (2006) 179-187.

[9] G. Christakos: Random Field Models in Earth Sciences. Academic Press, San Diego, CA, 1992.

[10] P. G. Ciarlet: The Finite Element Method for Elliptic Problems. NorthHolland, Amsterdam, 1978.

[11] G. Da Prato and J. Zabczyk: Stochastic Equations in Infinite Dimensions. Cambridge University Press, Cambridge, 1992.

[12] J. E. Dennis, Jr. and R. B. Schnabel. Numerical methods for unconstrained optimization and nonlinear equations. Classics in applied mathematics. SIAM, Philadelphia, PA, 1996. 
[13] Ph. Frauenfelder, Chr. Schwab and R. A. Todor: Finite elements for elliptic problems with stochastic coefficients. Comp. Meth. Appl. Mech. Engrg. 194 (2005) 205-228.

[14] T. Gerstner and M. Griebel: Numerical integration using sparse grids. Numer. Algorithms 18 (1998) 209-232.

[15] R. Ghanem and P. D. Spanos: Stochastic Finite Elements-A Spectral Approach. Springer-Verlag, Berlin, 1991.

[16] R. Ghanem: Stochastic finite elements for heterogeneous media with multiple random non-Gaussian properties. ASCE J. Engrg. Mech. 125 (1999) 24-40.

[17] T. Hida, H.-H. Kuo, J. Potthoff, and L. Streit: White Noise-An Infinite Dimensional Calculus. Kluwer, Dordrecht, 1993.

[18] H. Holden, B.Øksendal, J. Ubøe, and T.-S. Zhang: Stochastic Partial Differential Equations. Birkhäuser Verlag, Basel, 1996.

[19] S. Janson: Gaussian Hilbert Spaces. Cambridge University Press, Cambridge, 1997.

[20] M. Jardak, C.-H. Su, and G. E. Karniadakis: Spectral polynomial chaos solutions of the stochastic advection equation. SIAM J. Sci. Comput. 17 (2002) 319-338.

[21] H. Jeggle. Nichtlineare Funktionalanalysis. Teubner, Stuttgart, 1979.

[22] G. Kallianpur: Stochastic Filtering Theory. Springer-Verlag, Berlin, 1980.

[23] G. E. Karniadakis, C.-H. Sue, D. Xiu, D. Lucor, C. Schwab and R. A. Tudor: Generalized polynomial chaos solution for differential equations with random input. Research Report 2005-1, SAM, ETH Zürich, Zürich, 2005

[24] A. Keese: A review of recent developments in the numerical solution of stochastic PDEs (stochastic finite elements). Informatikbericht 2003-6, Institute of Scientific Computing, Department of Mathematics and Computer Science, Technische Universität Braunschweig, Brunswick, 2003. http://opus.tu-bs.de/opus/volltexte/2003/504/

[25] P. Krée and C. Soize: Mathematics of Random Phenomena. D. Reidel, Dordrecht, 1986. 
[26] O. P. Le Maître, H. N. Najm, R. G. Ghanem and O. M. Knio: Multiresolution analysis of Wiener-type uncertainty propagation schemes. J. Comp. Phys. 197 (2004) 502-531.

[27] P.-L. Lions and P. E. Souganidis: Fully nonlinear stochastic partial differential equations. C. R. Acad. Sci. Paris, Série I. 326 (1998) 10851092.

[28] P. Malliavin: Stochastic Analysis. Springer-Verlag, Berlin, 1997.

[29] H. Matthies and G. Strang. The solution of nonlinear finite element equations. Int. J. Numer. Methods Engrg., 14:1613-1626, 1979.

[30] H. G. Matthies, C. E. Brenner, C. G. Bucher, and C. Guedes Soares: Uncertainties in probabilistic numerical analysis of structures and solidsstochastic finite elements. Struct. Safety 19 (1997) 283-336.

[31] H. G. Matthies and C. G. Bucher: Finite elements for stochastic media problems. Comp. Meth. Appl. Mech. Engrg. 168 (1999) 3-17.

[32] H. G. Matthies and A. Keese: Galerkin methods for linear and nonlinear elliptic stochastic partial differential equations. Comp. Meth. Appl. Mech. Engrg. 194 (2005) 1295-1331.

[33] H. G. Matthies: Quantifying Uncertainty: Modern Computational Representation of Probability and Applications. In: A. Ibrahimbegović and I. Kožar (eds.), Extreme Man-Made and Natural Hazards in Dynamics of Structures. NATO-ARW series. Springer Verlag, Berlin, 2007.

[34] H. G. Matthies: Stochastic Finite Elements: Computational Approaches to Stochastic Partial Differential equations. Z. Angew. Math. Mech. 88 (2008) 849-873.

[35] H. G. Matthies and E. Zander: Solving stochastic systems with low-rank tensor compression. Submitted to Linear Algebra and its Applications. 2009 .

[36] F. Nobile, R. Tempone and C. G. Webster: Sparse grid stochastic collocation method for elliptic partial differential equations with random input data. SIAM J. Numer. Analysis 46 (2008) 2309-2345.

[37] F. Nobile, R. Tempone and C. G. Webster: An anisotropic sparse grid stochastic collocation method for partial differential equations with random input data. SIAM J. Numer. Analysis 46 (2008) 2411-2442. 
[38] E. Novak and K. Ritter: The curse of dimension and a universal method for numerical integration. pp. 177-188 in G. Nürnberger, J. W. Schmidt, and G. Walz (eds.), Multivariate Approximation and Splines, ISNM. Birkhäuser Verlag, Basel, 1997.

[39] J. T. Oden. Qualitative Methods in Nonlinear Mechanics. Prentice-Hall, Englewood Cliffs, NJ, 1986.

[40] K. Petras: Fast calculation of coefficients in the Smolyak algorithm. Numer. Algorithms 26 (2001) 93-109.

[41] L. J. Roman and M. Sarkis: Stochastic Galerkin Method for Elliptic SPDEs: A White Noise Approach. Discrete and Continuous Dynamical Systems - Series B 6 (2006) 941-955.

[42] Yu. Rozanov: Random Fields and Stochastic Partial Differential Equations. Kluwer, Dordrecht, 1996.

[43] G. I. Schuëller: A state-of-the-art report on computational stochastic mechanics. Prob. Engrg. Mech. 14 (1997) 197-321.

[44] G. I. Schuëller: Recent developments in structural computational stochastic mechanics. pp. 281-310 in B.H.V. Topping (ed.), Computational Mechanics for the Twenty-First Century. Saxe-Coburg Publications, Edinburgh, 2000.

[45] G. I. Schuëller and P. D. Spanos (eds.): Monte Carlo Simulation. Balkema, Rotterdam, 2001.

[46] I. E. Segal and R. A. Kunze: Integrals and Operators. Springer-Verlag, Berlin, 1978.

[47] G. Strang and G. J. Fix: An Analysis of the Finite Element Method. Wellesley-Cambridge Press, Wellesley, MA, 1988.

[48] B. Sudret and A. Der Kiureghian: Stochastic finite element methods and reliability. A state-of-the-art report. Report UCB/SEMM-2000/08, Department of Civil \& Environmental Engineering, University of California, Berkeley, CA, 2000.

[49] E. Ullmann: A Kronecker product preconditioner for stochastic Galerkin finite element discretizations. SIAM J. Sci. Comput. 32 (2010) 923-946.

[50] E. Vanmarcke: Random Fields: Analysis and Synthesis. The MIT Press, Cambridge, MA, 1988. 
[51] X. Wan and G. E. Karniadakis: An adaptive multi-element generalized polynomial chaos method for stochastic differential equations. J. Comp. Phys. 209 (2005) 617-642.

[52] D. Xiu and G. E. Karniadakis: Modeling uncertainty in steady state diffusion problems via generalized polynomial chaos. Comp. Meth. Appl. Mech. Engrg. 191 (2002) 4927-4948.

[53] X. F. Xu: A multiscale stochastic finite element method on elliptic problems involving uncertainties. Comp. Meth. Appl. Mech. Engrg. 196 (2007) 2723-2736.

[54] E. Zander and H. G. Matthies: Tensor product methods for stochastic problems. Proc. Appl. Math. Mech. 7 (2008) 2040067-2040068. 


\section{Technische Universität Braunschweig}

Informatik-Berichte ab Nr. 2008-06

2008-06 M. Broy, M. V. Cengarle, H. Grönniger B. Rumpe

2008-07 C. Basarke, C. Berger, K. Berger, K. Cornelsen, M. Doering J. Effertz, T. Form, T. Gülke, F. Graefe, P. Hecker, K. Homeier F. Klose, C. Lipski, M. Magnor, J. Morgenroth, T. Nothdurft, S. Ohl, F. Rauskolb, B. Rumpe, W. Schumacher, J. Wille, L. Wolf

2008-08 B. Rosic

2008-09 B. N. Khoromskij, A. Litvinenko, H. G. Matthies

2008-10 M. V. Cengarle, H. Grönniger B. Rumpe

2009-01 H. Giese, M. Huhn, U. Nickel, B. Schätz (Herausgeber)

2009-02 D. Jürgens

2009-03 O. Pajonk

2009-04 B. Sun, M. Lochau, P. Huhn, U. Goltz

2009-05 A. Rausch, U. Goltz, G. Engels, M. Goedicke, R. Reussner

2009-06 T. Müller, M. Lochau, S. Detering, F. Saust, H. Garbers, L. Märtin, T. Form, U. Goltz

2009-07 M. Huhn, C. Knieke

2010-01 A. Litvinenko and H. G. Matthies

2010-02 D. Grunwald, M. Lochau, E. Börger, U. Goltz

2010-03 M. Krosche, R. Niekamp

2011-01 L. Märtin, M. Schatalov, C. Knieke

2011-02 B. V. Rosić, A. Litvinenko, O. Pajonk, H. G. Matthies

2011-03 H. G. Matthies

2011-04 O. Pajonk, B. Rosić, A. Litvinenko, and H. G. Matthies

2011-05 H. G. Matthies
Modular Description of a Comprehensive Semantics Model for the UML (Version 2.0)

2007 DARPA Urban Challenge Team CarOLO Technical Paper

A Review of the Computational Stochastic Elastoplasticity

Application of Hierarchical Matrices for Computing the Karhunen-Loeve Expansion

System Model Semantics of Statecharts

Tagungsband des Dagstuhl-Workshops MBEES:

Modellbasierte Entwicklung eingebetteter Systeme V

Survey on Software Engineering for Scientific

Applications: Reuseable Software, Grid Computing and Application

Overview of System Identification with Focus on Inverse Modeling

Parameter Optimization of an Engine Control Unit using Genetic Algorithms

LaZuSo 2009: 1. Workshop für langlebige und zukunftsfähige Softwaresysteme 2009

Umsetzung eines modellbasierten durchgängigen Enwicklungsprozesses für AUTOSAR-Systeme mit integrierter Qualitätssicherung

Semantic Foundation and Validation of Live Activity Diagrams

Sparse data formats and efficient numerical methods for uncertainties quantification in numerical aerodynamics

An Abstract State Machine Model for the Generic Java Type System

Low-Rank Approximation in Spectral Stochastic Finite Element Method with Solution Space Adaption

Entwicklung und Erweiterung einer Werkzeugkette im Kontext von IT-Ökosystemen

Direct Bayesian update of polynomial chaos representations

White Noise Analysis for Stochastic Partial Differential Equations

A Deterministic Filter for non-Gaussian Bayesian Estimation

A Hitchhiker's Guide to Mathematical Notation and Definitions 